\title{
Experimental Study of Micro-EDM Precision Milling
}

\author{
Liaoyuan Zhang ${ }^{1, \text { a }}$, Kangle Ma ${ }^{1, b}$, Yue Geng ${ }^{1}$, Yan Zhao ${ }^{1}$ \\ ${ }^{1}$ School of Shenyang Li gong University, Shenyang, 110159, China; \\ a email: zly6217@163.com, bemail: 15904010298@163.com
}

Key works: EDM, Micro and Small hole machining, The machining accuracy, Orthogonal test Abstract: With the continuous development of aeronautical an space technologies, greater demands are being placed on the aviation and aerospace engine performance. The machining accuracy of fuel injection micro hole has great influence on engine performance. The machining accuracy of micro hole is closely related to flow rate and other physical parameters, so the research and analysis on processing high-accuracy micro hole are of great significance. The thesis researches on the inaccuracy of micro hole's machining straightness and cylindricity in the way of experimental study and analysis on micro EDM milling, optimization of electrical parameters, layered machining of tiny thickness and so on. The result shows that when the no-load voltage is $110 \mathrm{~V}$, the peak current is $70 \mathrm{~A}$, the pulse length is $4 \mu \mathrm{s}$, the interval of pulse is $90 \mu \mathrm{s}$, layered thickness is $0.15 \mathrm{~mm}$ and the discharge gap is $0.012 \mathrm{~mm}$, micro hole's milling gets the highest accuracy, and the range of dimensional accuracy processing is $0.005 \mathrm{~mm}$, the surface roughness is $0.427 \mu \mathrm{m}$.

\section{Introduction}

With the development of aerospace technology in difficult to machine materials nickel and Ti-based alloys, holes and great depth typical characteristics $0.2-0.4 \mathrm{~mm}$ in diameter are widely used in aerospace components. Micro EDM milling technology in processing of large aspect ratio fine grooves, cavities and other minor size has outstanding advantages. Typical applications is the fuel injection nozzle for enhance the accuracy requirements. The atomization degree depends on the flow rate, the diameter of the nozzle, the surface roughness and the surface texture of the nozzle, and the radius of the nozzle [1].FLUNT software was used to simulation that diesel nozzle single hole flow model parameters and the relationship between the dimensional accuracy of the injection hole [2]. The simulation model only changed the pore size. Pressure inlet pressure is $80 \mathrm{Mpa}$, and export atmospheric air pressure $0.1 \mathrm{Mpa}$. When the internal orifices roughness of $0.4 \mu \mathrm{m}$, the simulation of pores size of $\Phi 0.30 \mathrm{~mm}, \Phi 0.35 \mathrm{~mm}$ and $\Phi 0.40 \mathrm{~mm}$ nozzle exit at the traffic.

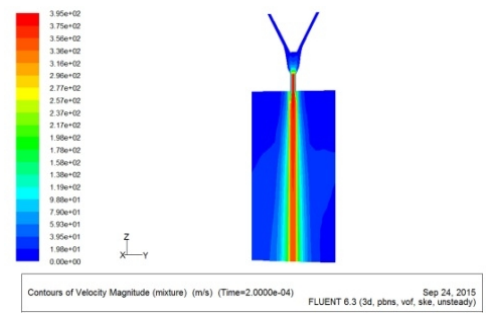

(a) (0.30mm, Ra0. $4 \mu \mathrm{m}$

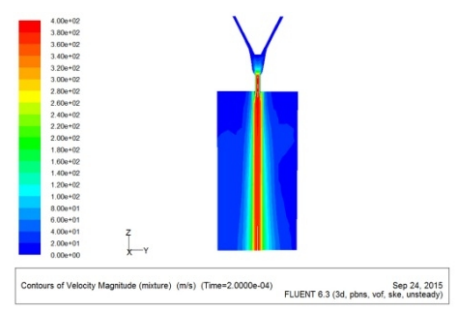

(b) $\Phi 0.35 \mathrm{~mm}$, Ra0. $4 \mu \mathrm{m}$

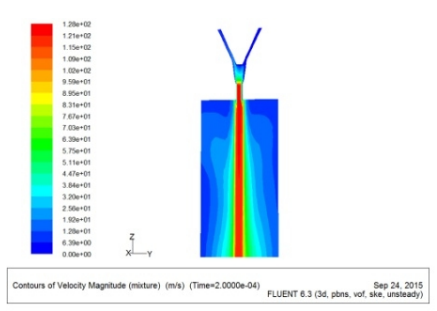

(c) $\Phi 0.40 \mathrm{~mm}, \mathrm{Ra} 0.4 \mu \mathrm{m}$

Fig.1. Fluent simulation of different aperture length

The orifice with $\Phi 0.30 \mathrm{~mm}$, with $\Phi 0.35 \mathrm{~mm}$ and $\Phi 0.40 \mathrm{~mm}$, the difference in size is $0.05 \mathrm{~mm}$, but the velocity distribution is significantly different. Different nozzle exit cross-section at the maximum flow rate, $\Phi 0.30 \mathrm{~mm}$ orifices flow rate of $395 \mathrm{~m} / \mathrm{s}, \Phi 0.35 \mathrm{~mm}$ orifices flow rate of $400 \mathrm{~m} / \mathrm{s}$, $\Phi 0.40 \mathrm{~mm}$ orifices flow rate of $328 \mathrm{~m} / \mathrm{s}$. In a predefined manufacturing tolerance range to reduce the 
hole diameter and increase the length to diameter ratio is development trend of fuel injection hole. Therefore, the study of micro EDM milling machining accuracy in the law are important to deep holes and deep grooves.

\section{U groove machining experiment}

\section{Experiment device}

Experimental apparatus use SX-100HPM micro EDM machine. It has a high-precision, high-speed and other characteristics; it is a dedicated micro-machining and micro fine piece of electrical discharge machining equipment. Processing equipment principle experiment shown in Figure 2.The parameters of machine tool as $\mathrm{X} / \mathrm{Y} / \mathrm{Z} / \mathrm{Z} 2$ axis travel distance is 250 $\times 150 \times 150 \times 150 \mathrm{~mm}$ : Z-axis feed rate is maximum $650 \mathrm{~mm} / \mathrm{min}$ : positioning accuracy is \pm $2 \mathrm{~mm}$. Thereby improve processing efficiency, improve the processing quality. In the process of micro deep hole and shaped hole, it can ensure the accuracy of the hole, the shape precision and the size of the hole.

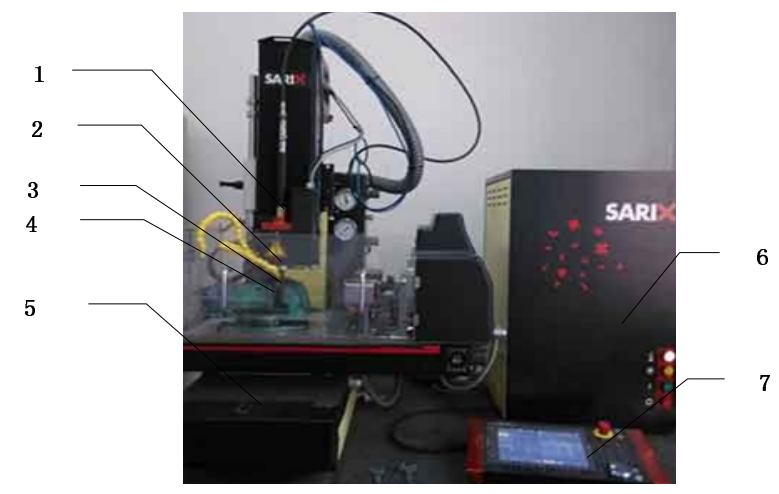

Fig.2.The principle diagram of the machine tool

1:High-speed spindle 2: Working liquid 3: Electrode 4: Artifacts 5: Machine tool lathe bed 6: Pulse power 7: Operation panel

\section{a) The basic parameters}

Study, negative working, carbide electrode material, the electrode diameter is $0.4 \mathrm{~mm}$, the machine speed is 720rmp, work-piece material is 45 steel, medium to aviation kerosene. U-shaped groove machining depth is $4.5 \mathrm{~mm}$, width is $0.4 \mathrm{~mm}$. The main laboratory equipment are SX-100HPM micro EDM machine, optical microscope $40 * 5000 *$, CCD500 *, Bruker white light interferometer.

\section{b) Experimental principle}

Electric discharge milling method is divided into electrode side discharge machining and electrode bottom discharge machining, as shown in figure 3. Because of large-area electrode side discharge machining processing, to make the electrode loss, increase precision machining size error. Further, since the sharp edge of the discharge electrode tip, loss of fillet formation [3]. Experiment uses a small thickness layers, reciprocating milling processing methods, as shown in figure4. The electrode loss is bound to the end surface, and the influence of the electrode on the loss of the diameter and the discharge of the fillet is reduced to the machining precision of the $\mathrm{U}$ groove. Figure 5 is the intention of the length direction of layered milling electrode movement and sharp loss. Figure 5 is a schematic diagram of the reciprocating movement and loss of the electrodes in the diameter direction. 


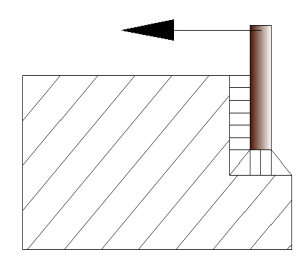

(a)The side discharge machining

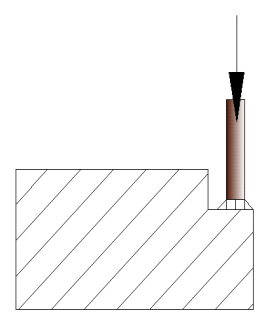

(b) bottom discharge

Fig.3.Edm milling method
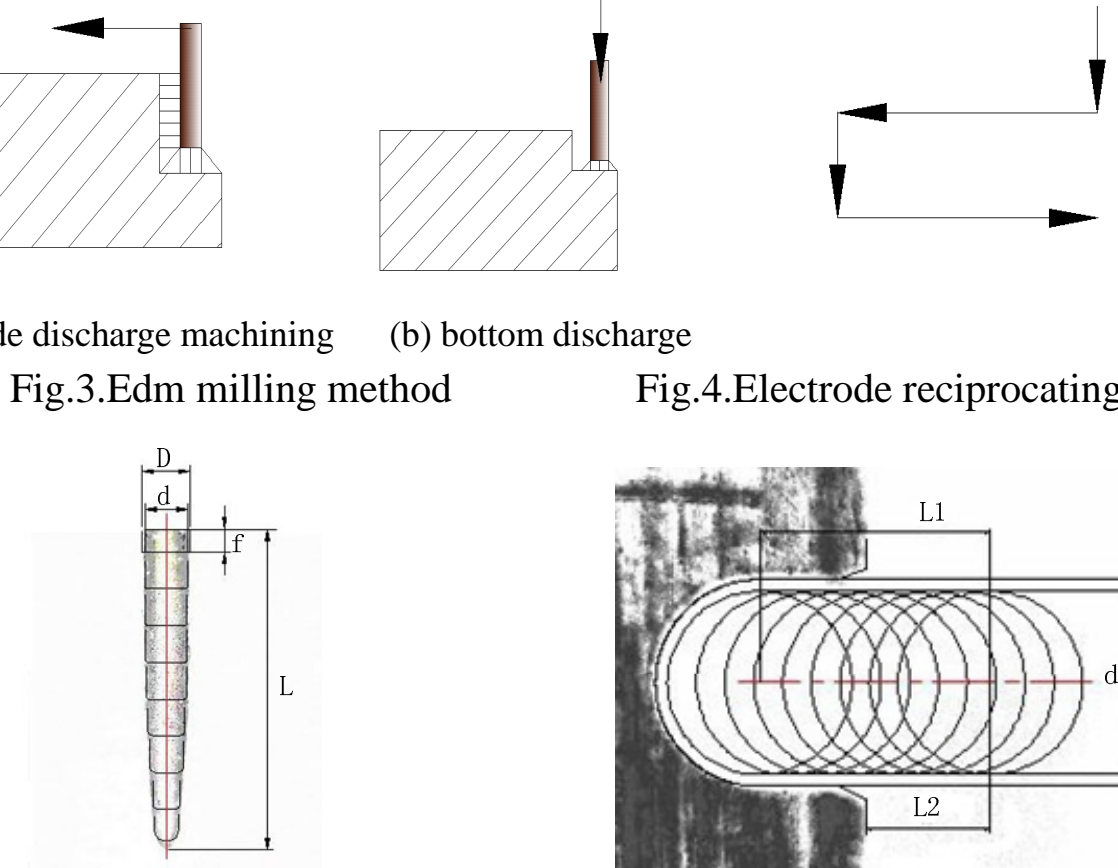

Fig.4.Electrode reciprocating motion track

(a)The length of the direction

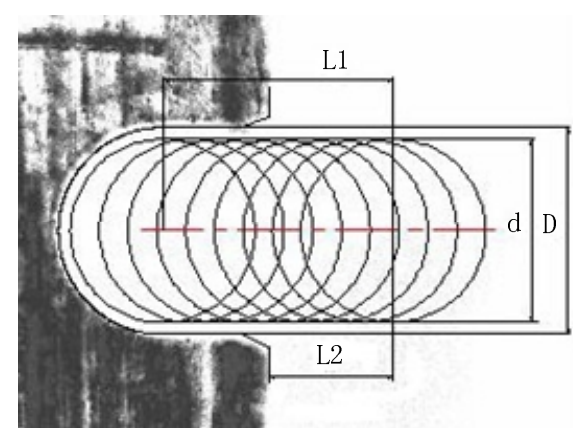

(b) The diameter of the direction

Fig.5.The principle diagram of the electrode wire feed

Figure 5 (a) d is the wire diameter (same as Figure b), D for the actual processing of the U groove width (with Figure b), $\mathrm{f}$ is the feed rate of the layer thickness; L is the total length of the groove machining $\mathrm{U}$. In the process of electrode wire slicing, the loss of the electrode wire in the radial direction is small. In the length direction, the tip discharge principle, and gradually formed a round, so the lower groove U formed V sharp. Figure 5 (b) is a boundary contour map of the diameter.

\section{The experimental results and discussion}

The orthogonal analysis, include electrical parameters and results of choice, as in table 1. Use five factors and four levels, we carried out 16 experiments. In order to reduce the interference of other factors in the process of the experiment, $3 \mathrm{U}$ - shaped groove in each experiment. Scaling size using an optical microscope, the average taken as a result of the experiment. Among them, the relationship between the aperture after the finished aperture gap between the electrode diameter through mathematical calculations, is $\delta=(\mathrm{D}-\mathrm{d}) / 2$. 
Table 1 The experimental data and results

\begin{tabular}{|c|c|c|c|c|c|c|}
\hline & $\begin{array}{c}\text { Voltage } \\
\text { U/V }\end{array}$ & I/A & $\mathrm{T} / \mu \mathrm{s}$ & $\begin{array}{c}\text { Between the } \\
\text { pulse } \\
\mathrm{T} / \mu \mathrm{s}\end{array}$ & $\begin{array}{c}\text { Thickness of } \\
\text { the layered } \\
\text { mm }\end{array}$ & Aperture gap \\
\hline 1 & 60 & 5 & 1 & 4 & 0.09 & 0.024 \\
\hline 2 & 60 & 20 & 4 & 30 & 0.12 & 0.028 \\
\hline 3 & 60 & 50 & 5 & 90 & 0.15 & 0.014 \\
\hline 4 & 60 & 70 & 7 & 120 & 0.18 & 0.009 \\
\hline 5 & 80 & 5 & 4 & 90 & 0.18 & 0.019 \\
\hline 6 & 80 & 20 & 1 & 120 & 0.15 & 0.024 \\
\hline 7 & 80 & 50 & 7 & 4 & 0.12 & 0.032 \\
\hline 8 & 80 & 70 & 5 & 30 & 0.09 & 0.021 \\
\hline 9 & 110 & 5 & 5 & 120 & 0.12 & 0.023 \\
\hline 10 & 110 & 20 & 7 & 90 & 0.09 & 0.018 \\
\hline 11 & 110 & 50 & 1 & 30 & 0.18 & 0.036 \\
\hline 12 & 110 & 70 & 4 & 4 & 0.15 & 0.015 \\
\hline 13 & 160 & 5 & 7 & 30 & 0.15 & 0.022 \\
\hline 14 & 160 & 20 & 5 & 4 & 0.18 & 0.012 \\
\hline 15 & 160 & 50 & 4 & 120 & 0.09 & 0.033 \\
\hline 16 & 160 & 70 & 1 & 90 & 0.12 & 0.035 \\
\hline t1 & 0.019 & 0.022 & 0.030 & 0.021 & 0.024 & \\
\hline $\mathrm{t} 2$ & 0.024 & 0.021 & 0.024 & 0.027 & 0.029 & \\
\hline t3 & 0.023 & 0.029 & 0.018 & 0.022 & 0.019 & \\
\hline t4 & 0.026 & 0.020 & 0.020 & 0.022 & 0.019 & \\
\hline $\mathrm{R}$ & 0.0075 & 0.009 & 0.012 & 0.006 & 0.010 & \\
\hline
\end{tabular}

By the magnitude of the $\mathrm{R}$ in the upper table, we can determine the parameters of the single process parameters optimization of the primary and secondary order. The greater the $\mathrm{R}$ value, the greater the impact of the experimental results, from the big to the small :pulse width of time, the thickness of the layered, peak current, Open circuit voltage, Open circuit voltage. Pulse width influence value of 0.012 , layering thickness of 0.010 , the peak current is 0.075 , the no-load voltage is 0.009 , and the minimum pulse interval is 0.006 .U-shaped slot radial dimensions mainly by electro discharge machining, the machining gap between the wire electrode and workpiece.

\section{Dimensional accuracy experiment analysis}

In the measurement and judgment of dimensional accuracy, the usual way is to use micro vision measurement method. Considering the radial and length size of the $U$ groove, the accuracy of the experimental results is better. The experimental results were treated by the method of gray boundary treatment.

Because the experimental processing part is small, the direct measurement, the accuracy has the influence. CCD images were collected and processed using the MATLAB edge gray level fitting method. MATLAB converts color images into grayscale images. The edge detection algorithm based on MATLAB. First extraction reflect changes in the gray edge points, the edge point set again excluded certain border points or to fill boundary discontinuities, and connect the edges into a complete line. The CANNY operator is used to detect the strong and weak edges, and the weak edges are included in the output images when the weak edges and the edges are connected. Canny operator also is not susceptible to noise interference, able to detect true weak edges [5]. Its 
significance lies in processing after a little cutting adhesion in the u-shaped slot wall, also can make the processed surface precision of the output, improve the dimensional accuracy. Extract the edge of the curve is in the image in the matrix expression of the determination of the only. MATLAB makes the image signal into digital signal, using mathematical formula. Computer program automatically judgment standard to distinguish the edge line, avoid deviation due to conscious control.

The fifth group and the sixth groups of radial gray curve respectively, as shown in figure 6 . The boundary of the CANNY operator handled, as the figure 7 The fifth and sixth groups of gray level of function curve under a coordinate, under the same coordinate figure 8 (a).The deviation is small, local amplification figure to 8(b).

The dimensional accuracy of the two methods radial contrast.MATLAB gray processing of experimental data, than the actual measurement of experimental data, the accuracy of numerical raised a decimal point, the number of more accurate. Its conclusions are consistent, electric parameters affect the pulse width is the largest.

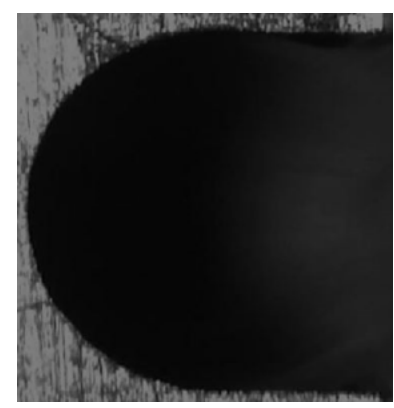

(a) The fifth

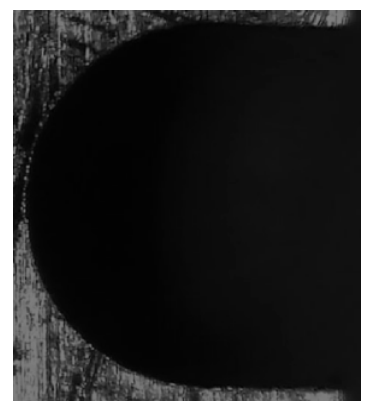

(b) The sixth

Fig.6. Grayscale

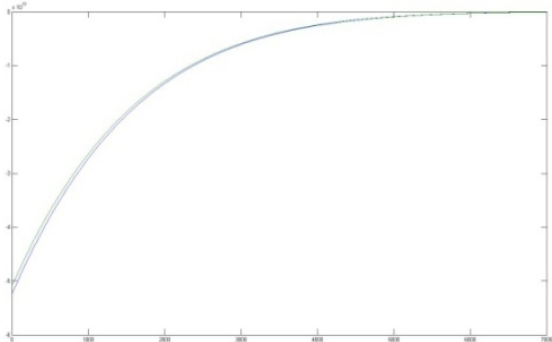

(a) The radial function fitting

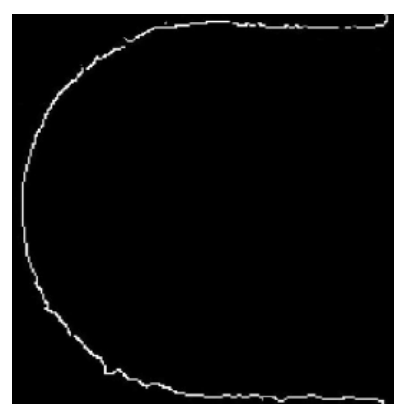

(a) The fifth

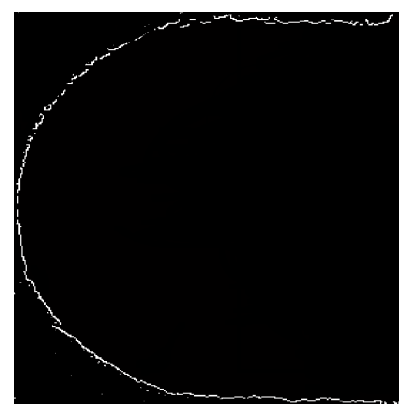

(b) The sixth

Fig.7.Canny operator edge image

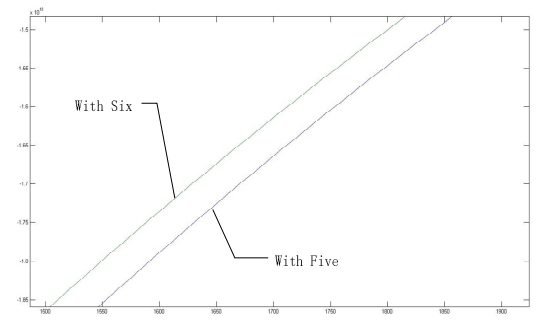

(b) Enlarge figure

Fig.8The radial edge function comparison results

\section{The surface quality analysis}

The surface roughness is one of the index reflect the workpiece surface quality. Spark discharge machining is the electrode discharge channel transfer of material forming process of electrode surface topography [6]. Every effective pulse discharge form surface depressions, overlapping of single pulse discharge crater formed eventually surface topography. So the shape of the workpiece surface characteristics and the shape of the surface roughness is made up of single pulse discharge crater size and the degree of overlap [7]. In view of the u-shaped slot internal surface quality, the experiment using BRUKER white light interferometer, the measuring line. White light interferometer with non-contact measurement, the use of light in different surface reflection after the formation of the interference fringes is analyzed. In wavelength measurement optical path difference, its measurement precision is extremely high. The surface of the u-shaped groove profile processing pit is figure 9(a). U-shaped groove profile roughness of three-dimensional contour shape, color change on behalf of its relative to the same horizontal plane height change 
value is $9(\mathrm{~b})$. Measuring results output is figure 9(c).

According to the data measured roughness $\mathrm{Ra}$ and electrical parameters of the scope of the conclusion. As the current increases, the Ra increase, But when the current reaches a certain value, then the Ra no longer increase but stable. Pulse interval to the influence of surface roughness, when the pulse width of time must be the smaller the pulse interval, reduces Ra. But certain conditions, the increase of pulse width of time, $\mathrm{Ra}$, when the pulse interval increases to a certain value, the $\mathrm{Ra}$ decreases with the increase of the pulse interval. Due to the smaller the pulse interval, the shorter the single pulse discharge cycle, pulse discharge increased frequency, pulse refinement, makes the single pulse discharge energy is reduced, and then lower the Ra.

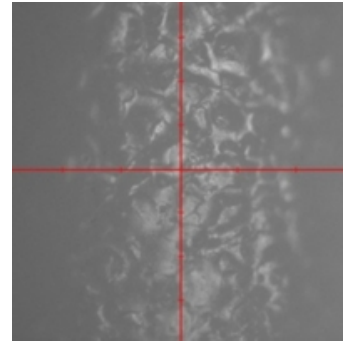

(a) $\mathrm{U}$ groove machining surface

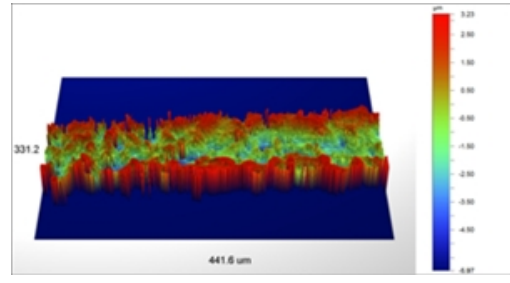

(b) Three-dimensional contour roughness

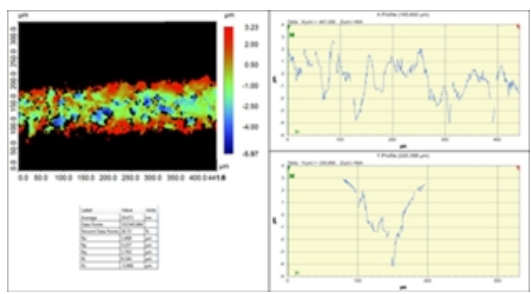

(c) Roughness values

Fig.9. BRUKER white light interferometer roughness measurement

\section{Conclusion}

The influence of pulse width of time for processing size is the largest. Within a certain range, with the increase of pulse width of time, dimensional accuracy improved. Experiment, layering thickness of dimensional precision, first with the increase of thickness of layered precision fall first, after the increase with the increase of layer thickness accuracy. Peak current on the dimensional accuracy, within a certain range influence line for serrated, 50A is the best current.

In this experiment to get the optimal parameters for voltage $110 \mathrm{~V}$, current is $50 \mathrm{~A}$, including pulse width is $4 \mu \mathrm{s}$, pulse is $90 \mu \mathrm{s}$, including s processing dimensional accuracy and surface quality of the best.

\section{References}

[1] Wu Zhengyv. Nozzle wall quality of research on the effects of emulsified diesel engine performance [D] Taiyuan; Taiyuan institute of technology, 2013

[2] Li Ying. Diesel engine fuel injection spray characteristics and influence factors analysis of theoretical research [D]; Nanjing; Nanjing institute of technology

[3] Cui Jing, Li Yong, Xiong Ying. One kind of high aspect ratio fine groove of EDM [J].Journal of Beijing industrial, 2010, 36(3):289-293

[4]Kong Deying, The engine combustion chamber nozzle Angle of oil mist image simulation experiment research [D].Xi'an, Northwestern polytechnic, 2007

[5]Liu Jingchun, Zhao Jiaqi, Zhao Wansheng. Special processing [M].Beijing, Mechanical industry press.1999

[6] Y.H. Guu, Max Ti-Kuang Hou. Effect of machining parameters on surface textures in EDM of Fe-Mn-Al alloy. In: Materials Science and Engineering A 2007, 466: 61-67. 